\title{
HERSCHEL* FAR-INFRARED SPECTROSCOPY OF THE GALACTIC CENTER. HOT MOLECULAR GAS: SHOCKS VERSUS RADIATION NEAR Sgr A*
}

\author{
Javier R. Goicoechea ${ }^{1}$, M. Etxaluze ${ }^{1}$, J. Cernicharo ${ }^{1}$, M. Gerin ${ }^{2}$, D. A. Neufeld ${ }^{3}$, A. Contursi ${ }^{4}$, T. A. Bell ${ }^{1}$,

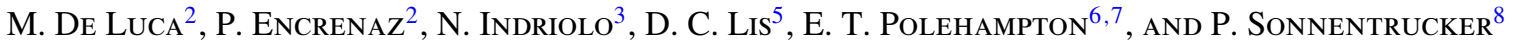 \\ ${ }^{1}$ Departamento de Astrofísica, Centro de Astrobiología, CSIC-INTA, Carretera de Ajalvir, Km 4, Torrejón de Ardoz, \\ E-28850 Madrid, Spain; jr.goicoechea@cab.inta-csic.es \\ ${ }^{2}$ LERMA, UMR 8112 du CNRS, Observatoire de Paris, École Normale Supérieure, France \\ ${ }^{3}$ The Johns Hopkins University, Baltimore, MD 21218, USA \\ ${ }^{4}$ Max-Planck-Institut für extraterrestrische Physik (MPE), Postfach 1312, D-85741 Garching, Germany \\ ${ }^{5}$ California Institute of Technology, Pasadena, CA 91125, USA \\ ${ }^{6}$ RAL Space, Rutherford Appleton Laboratory, Chilton, Didcot, Oxfordshire OX11 0QX, UK \\ ${ }^{7}$ Institute for Space Imaging Science, University of Lethbridge, 4401 University Drive, Lethbridge, Alberta T1J 1B1, Canada \\ ${ }^{8}$ Space Telescope Science Institute, Baltimore, MD 21218, USA \\ Received 2013 January 26; accepted 2013 March 28; published 2013 May 7
}

\begin{abstract}
We present a $\sim 52-671 \mu \mathrm{m}$ spectral scan toward Sgr A* taken with the PACS and SPIRE spectrometers on board Herschel. The achieved angular resolution allows us to separate, for the first time at far-IR wavelengths, the emission toward the central cavity (gas in the inner central parsec of the galaxy) from that of the surrounding circumnuclear disk. The spectrum toward Sgr $\mathrm{A}^{*}$ is dominated by strong [O III], [O I], [C II], [N III], [N II], and [C I] fine-structure lines (in decreasing order of luminosity) arising in gas irradiated by UV photons from the central stellar cluster. In addition, rotationally excited lines of ${ }^{12} \mathrm{CO}$ (from $J=4-3$ to $24-23$ ), ${ }^{13} \mathrm{CO}, \mathrm{H}_{2} \mathrm{O}, \mathrm{OH}, \mathrm{H}_{3} \mathrm{O}^{+}, \mathrm{HCO}^{+}$, and $\mathrm{HCN}$, as well as ground-state absorption lines of $\mathrm{OH}^{+}, \mathrm{H}_{2} \mathrm{O}^{+}, \mathrm{H}_{3} \mathrm{O}^{+}, \mathrm{CH}^{+}, \mathrm{H}_{2} \mathrm{O}, \mathrm{OH}, \mathrm{HF}, \mathrm{CH}$, and $\mathrm{NH}$ are detected. The excitation of the ${ }^{12} \mathrm{CO}$ ladder is consistent with a hot isothermal component at $T_{\mathrm{k}} \simeq 10^{3.1} \mathrm{~K}$ and $n\left(\mathrm{H}_{2}\right) \lesssim 10^{4} \mathrm{~cm}^{-3}$. It is also consistent with a distribution of temperature components at higher density with most $\mathrm{CO}$ at $T_{\mathrm{k}} \lesssim 300 \mathrm{~K}$. The detected molecular features suggest that, at present, neither very enhanced X-ray nor cosmic-ray fluxes play a dominant role in the heating of the hot molecular gas. The hot $\mathrm{CO}$ component (either the bulk of the CO column or just a small fraction depending on the above scenario) results from a combination of UV- and shock-driven heating. If irradiated dense clumps/clouds do not exist, shocks likely dominate the heating of the hot molecular gas. This is consistent with the high-velocity gas detected toward Sgr A*.
\end{abstract}

Key words: black hole physics - Galaxy: center - infrared: ISM - ISM: molecules - shock waves

Online-only material: color figures

\section{INTRODUCTION}

The Galactic center (GC), in particular the interstellar material in the immediate vicinity $(<5 \mathrm{pc})$ of the central black hole, represents a unique environment for our understanding of galactic nuclei and galaxy evolution. At a distance $d=8.0 \pm 0.5 \mathrm{kpc}$ (Reid 1993), the nucleus of our galaxy is a few hundred times closer than the nearest active galactic nuclei (AGNs), thus allowing high spatial resolution studies. The distribution of gas and dust toward the GC (Genzel et al. 2010) consists of a central cavity of radius $\sim 1.5 \mathrm{pc}$ containing warm dust and gas heated and ionized by the central cluster of massive stars orbiting close to the black hole (Sgr A* radio source position). Some of the ionized gas streamers (the "mini-spiral") bring material close to the very center (Yusef-Zadeh \& Morris 1987; see Figure 1).

Between $\sim 1.5 \mathrm{pc}$ and $\sim 5 \mathrm{pc}$, a disk of denser molecular gas exists (the circumnuclear disk or CND; Guesten et al. 1987). However, its density is not well constrained $\left(\sim 10^{4}-10^{8} \mathrm{~cm}^{-3}\right)$ and it is not yet clear whether all the material in the CND is stable against the strong tidal forces in the region or has a more transient nature (Bradford et al. 2005; Montero-Castaño et al. 2009; Requena-Torres et al. 2012). The molecular gas toward the central cavity has been less studied, first because high angular resolution is required to separate the different components and

\footnotetext{
* Herschel is an ESA space observatory with science instruments provided by European-led Principal Investigator consortia and with important participation from NASA.
}

also because column densities are inevitably lower and emission lines are intrinsically weak. Nevertheless, the detection of $\mathrm{CO}$ rovibrational lines in absorption (Moneti et al. 2001) and of broad $\mathrm{NH}_{3}(6,6)$ emission lines close to Sgr A* (Herrnstein \& Ho 2002) suggests that hot molecular gas must exist interior to the CND.

Owing to the lower dust extinction at far-IR wavelengths and because of the strong emission from the interstellar component related to AGN and star formation activity, the relevance of farIR spectroscopy to characterize extragalactic nuclei has notably increased (e.g., van der Werf et al. 2010). The far-IR spectrum of the Milky Way's nucleus is obviously a key template.

The angular resolution achieved by the Herschel Space Observatory $\left(\sim 10^{\prime \prime}-40^{\prime \prime}\right.$; Pilbratt et al. 2010$)$ allows us to separate the emission of the central cavity from the CND. In this Letter we present initial results from a Herschel far-IR spectroscopic study of the GC. We present the complete PACS (Poglitsch et al. 2010) and SPIRE (Griffin et al. 2010) spectra toward Sgr $\mathrm{A}^{*}$ that are part of the PRISMAS and SPECHIS Guaranteed-Time Programs. We discuss the properties and possible origin of the atomic and of the hot molecular gas.

\section{OBSERVATIONS AND DATA REDUCTION}

PACS spectra between $\sim 52$ and $\sim 190 \mu$ m were obtained during 2011 March and 2012 March. The PACS spectrometer provides 25 spectra over a $47^{\prime \prime} \times 47^{\prime \prime}$ field of view (FoV) resolved in $5 \times 5$ "spaxels," each with a size of $\sim 9$ ". 4 . The resolving power 

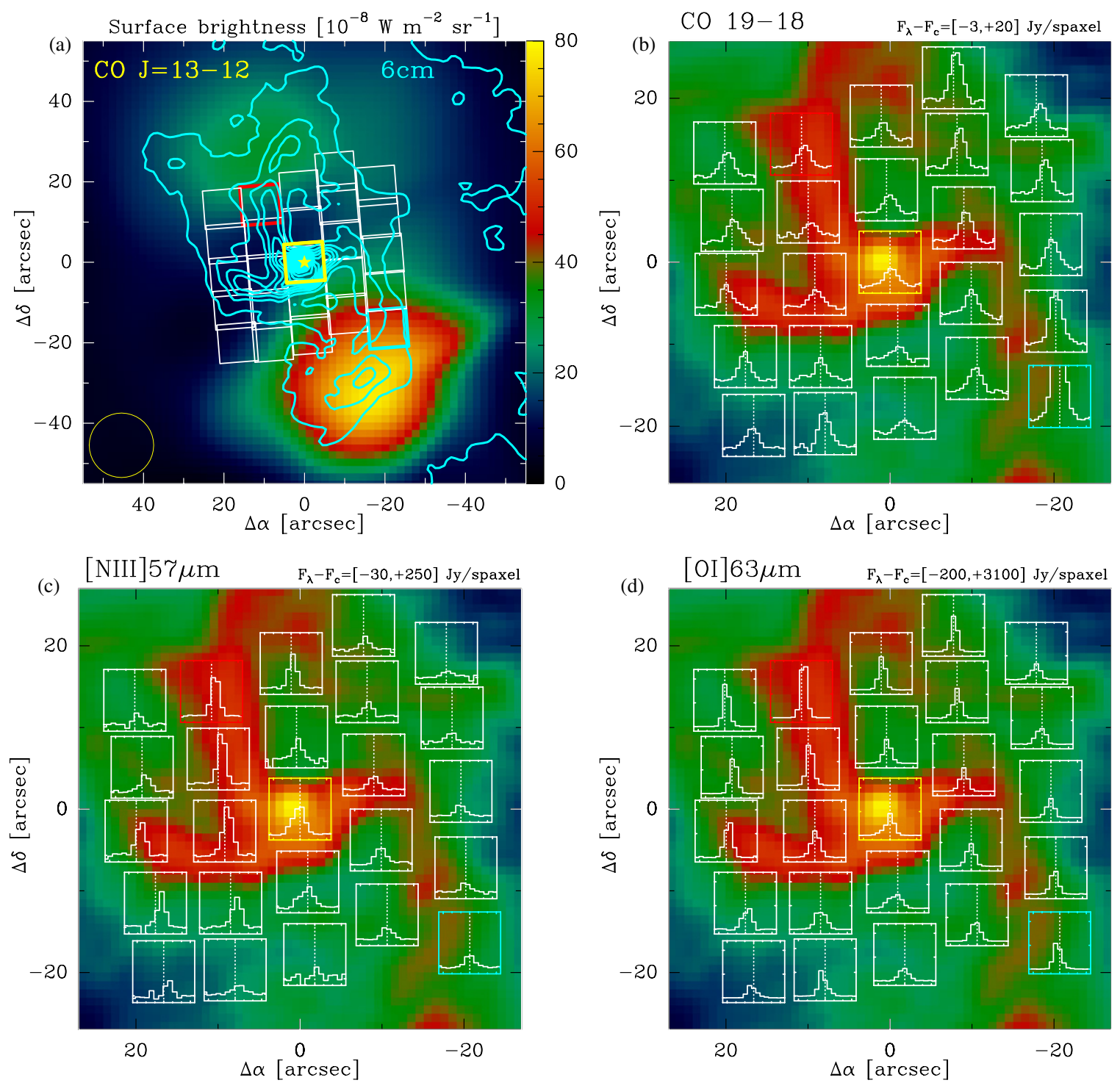

Figure 1. Panel (a): SPIRE-FTS ${ }^{12} \mathrm{CO} J=13-12$ sparse-sampling map of the GC and VLA $6 \mathrm{~cm}$ radio continuum showing ionized gas in the mini-spiral (cyan contours; Yusef-Zadeh \& Morris 1987). Sgr A* is marked with a star. The PACS footprint is overplotted. Panels (b), (c), and (d): PACS continuum-subtracted maps for the CO $J=19-18(137.196 \mu \mathrm{m})$, [N III] $57.317 \mu \mathrm{m}$, and [O I] $63.183 \mu \mathrm{m}$ lines. The center of each spaxel corresponds to its offset position with respect to Sgr $\mathrm{A}^{*}$. The $X$-axis represents the -700 to $+700 \mathrm{~km} \mathrm{~s}^{-1}$ velocity scale. The line flux scale ( $Y$-axis) is shown in each map. The $6 \mathrm{~cm}$ radio continuum image is shown in the background.

(A color version of this figure is available in the online journal.)

varies between $R=\lambda / \Delta \lambda \simeq 1000$ at $\sim 100 \mu \mathrm{m}$ and $R \simeq 5000$ at $\sim 70 \mu \mathrm{m}$. The central spaxel was centered on $\operatorname{Sgr~A}^{*}\left(\alpha_{2000}\right.$ : $\left.17^{\mathrm{h}} 45^{\mathrm{m}} 40^{\mathrm{s}} .04, \delta_{2000}:-29^{\circ} 00^{\prime} 28^{\prime \prime} .19\right)$. The observations were carried out in the "unchopped" mode (IDs 1342217802 and 342242442). Background subtraction was achieved by removing the telescope background spectrum measured on a distant offposition. The observing time was $\sim 2.4 \mathrm{hr}$. The measured width of the point-spread function is relatively constant for $\lambda \lesssim 100 \mu \mathrm{m}(\sim$ spaxel size $)$ but increases at longer wavelengths. In particular, only $\simeq 40 \%$ of a point source emission would fall in the central spaxel at $\simeq 190 \mu \mathrm{m}$. Therefore, owing to the extended nature of the emission, the flux measured by a single spaxel cannot be used individually. Instead, we added the $3 \times 3$ central spaxels $\left(\sim 30^{\prime \prime} \times 30^{\prime \prime}\right)$.

SPIRE-FTS observations between $\sim 194$ and $\sim 671 \mu \mathrm{m}$ were obtained during 2011 February (ID1342214845). The SPIRE-FTS uses two bolometer arrays covering the 194-313 $\mu \mathrm{m}$ and 303-671 $\mu \mathrm{m}$ bands at $0.04 \mathrm{~cm}^{-1}$ resolu- tion $(R \simeq 500-1000)$. The 2 arrays contain 19 and 37 detectors separated by $\sim 2$ beams ( $51^{\prime \prime}$ and $33^{\prime \prime}$, respectively). The unvignetted $\mathrm{FoV}$ is $\sim 2^{\prime}$. The observing time was $798 \mathrm{~s}$. The Herschel data were processed with HIPE 9.2. Table 1 summarizes the atomic and CO line intensities obtained in a $\sim 30^{\prime \prime} \times$ $30^{\prime \prime}$ aperture.

\section{RESULTS: SPECTROSCOPY}

Figure 1(a) shows the CO $J=13-12$ line intensity map of the central $\sim 4 \mathrm{pc}$ of the Galaxy displaying extended excited $\mathrm{CO}$ emission that peaks toward the northern and southern lobes of the CND (hereafter $N$-CND and $S$-CND). Figures 1(b)-(d) show the CO $J=19-18$, [N III] 57, and [O I] $63 \mu \mathrm{m}$ line spectral maps, respectively.

Figure 2 shows the complete $\sim 52-671 \mu \mathrm{m}$ spectrum toward Sgr A* (black curves) and toward a bright position at the inner edge of the $S$-CND, $\sim 1 \mathrm{pc}$ from Sgr A* (only for PACS; gray 

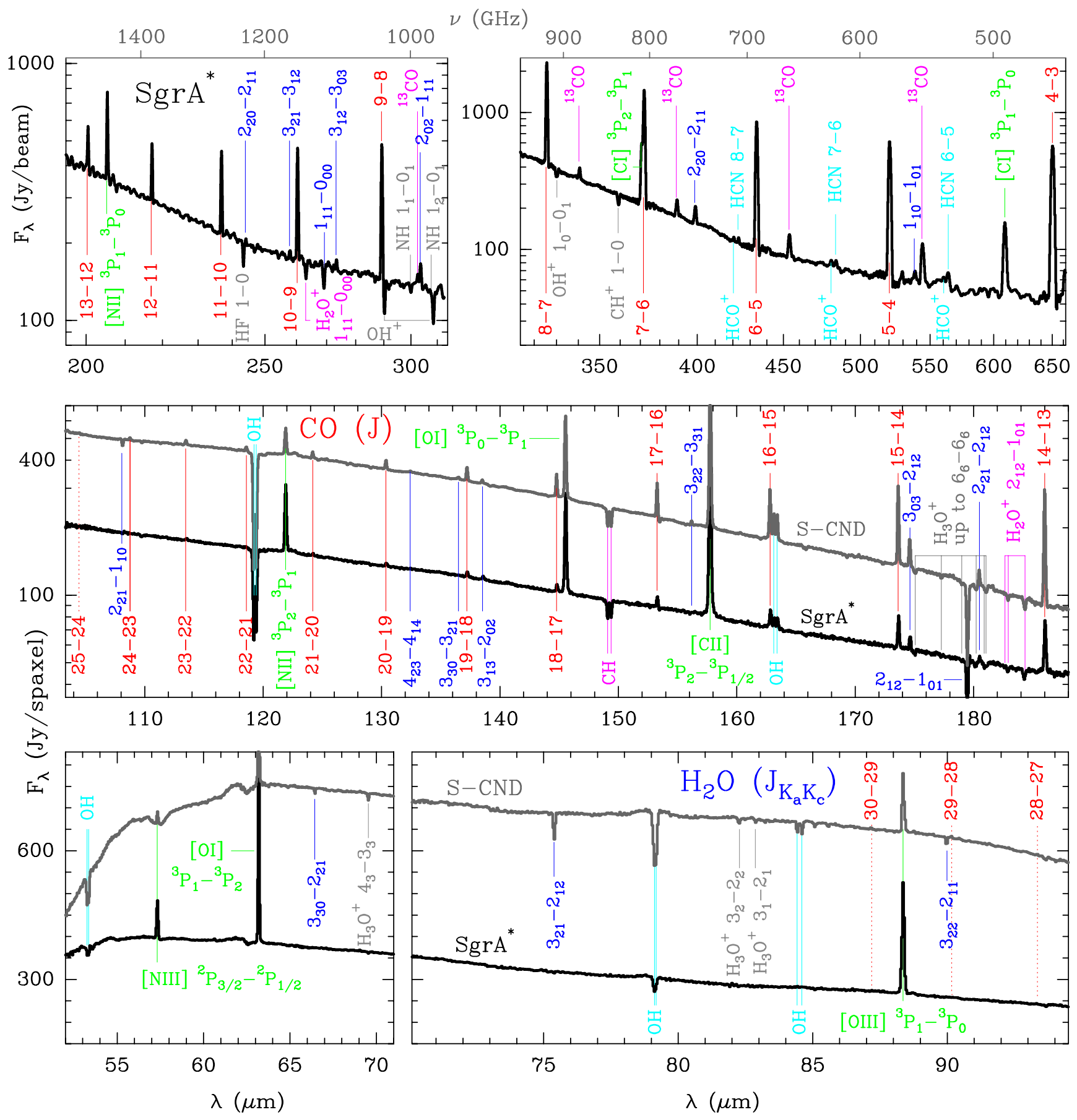

Figure 2. Top panel: SPIRE-FTS spectrum toward Sgr A*. Middle and bottom panels: PACS spectra toward Sgr A* (black curves; yellow spaxel in Figure 1(a)) and also toward the $S$-CND (gray curves; blue spaxel in Figure 1(a)). Flux density units are Jy spaxel ${ }^{-1}$ for PACS and Jy beam ${ }^{-1}$ for SPIRE.

(A color version of this figure is available in the online journal.)

curves). The far-IR spectrum toward Sgr A* is dominated by strong emission from atomic fine-structure lines ([O III], [O I], [C II], [N III], [N II], and [C I] ), high- $J$ CO rotational lines (up to $J=24-23$ toward the central cavity and up to $J=30-29$ in the $\mathrm{CND}$ ), mid- $\mathrm{J} \mathrm{HCO}^{+}$and $\mathrm{HCN}$ emission lines, and ground-state absorption lines from light hydrides $\left(\mathrm{OH}^{+}, \mathrm{H}_{2} \mathrm{O}^{+}, \mathrm{H}_{3} \mathrm{O}^{+}, \mathrm{CH}^{+}\right.$, $\mathrm{HF}, \mathrm{CH}, \mathrm{NH}, \mathrm{OH}$, and $\mathrm{H}_{2} \mathrm{O}$ ). Among the molecular ions, only $\mathrm{H}_{3} \mathrm{O}^{+}$shows absorption lines from excited levels (metastable levels up to $J_{K}=6_{6}$ toward the central cavity). The detection of rotationally excited lines from $\mathrm{H}_{2} \mathrm{O}, \mathrm{OH}$, and $\mathrm{H}_{3} \mathrm{O}^{+}$in absorption suggests that a non-negligible fraction of excited molecular gas resides at relatively low densities.
Owing to the $A_{V} \simeq 30$ mag of extinction toward the GC (Genzel et al. 2010), in the following discussion we correct all line intensities $\left(I_{0}=C_{\lambda} I_{\text {obs }}\right)$ using an extrapolation of the midIR extinction law derived by Lutz (1999) for Sgr A*. These corrections are $<15 \%$ in the far-IR (Table 1 ). The corrected line luminosities in the inner $\sim 30^{\prime \prime} \times 30^{\prime \prime}(\sim 0.6 \mathrm{pc}$ in radius $)$

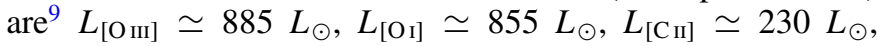
$L_{[\mathrm{N} \mathrm{III}]} \simeq 130 L_{\odot}, L_{[\mathrm{NII}]} \simeq 120 L_{\odot}, L_{{ }^{12} \mathrm{CO}} \simeq 125 L_{\odot}$, $L^{1{ }^{3} \mathrm{CO}} \simeq 4 L_{\odot}$, and $L_{\left[\mathrm{C}_{\mathrm{I}]}\right]} \simeq 6 L_{\odot}($ adopting $d=8 \mathrm{kpc})$.

\footnotetext{
9 We obtain $L_{\mathrm{FIR}}(50-1000 \mu \mathrm{m}) \simeq 10^{5.1} L_{\odot}$ using Herschel photometric data
} from Etxaluze et al. (2011). 
Table 1

Atomic and CO Line Intensities Toward Sgr A*

\begin{tabular}{|c|c|c|c|c|c|}
\hline Species & Transition & $\begin{array}{c}\lambda \\
(\mu \mathrm{m})\end{array}$ & $\begin{array}{c}E_{\mathrm{u}} / k \\
(\mathrm{~K})\end{array}$ & $I_{\mathrm{obs}}{ }^{\mathrm{a}}$ & $C_{\lambda}{ }^{\mathrm{b}}$ \\
\hline [O III] & ${ }^{3} P_{2}-{ }^{3} P_{1}$ & 51.815 & 441 & $1.52 \mathrm{E}-05^{\mathrm{c}}$ & 1.152 \\
\hline$[\mathrm{N}$ III $]$ & ${ }^{2} P_{3 / 2}-{ }^{2} P_{1 / 2}$ & 57.317 & 251 & $2.69 \mathrm{E}-06$ & 1.132 \\
\hline$\left[\mathrm{O}_{\mathrm{I}}\right]$ & ${ }^{3} P_{1}-{ }^{3} P_{2}$ & 63.184 & 228 & $1.62 \mathrm{E}-05$ & 1.116 \\
\hline [O III] & ${ }^{3} P_{1}-{ }^{3} P_{0}$ & 88.356 & 163 & $3.30 \mathrm{E}-06$ & 1.076 \\
\hline$[\mathrm{N}$ II $]$ & ${ }^{3} P_{2}-{ }^{3} P_{1}$ & 121.898 & 188 & $2.21 \mathrm{E}-06$ & 1.051 \\
\hline$\left[\mathrm{O}_{\mathrm{I}}\right]$ & ${ }^{3} P_{0}-{ }^{3} P_{1}$ & 145.525 & 327 & $2.11 \mathrm{E}-06$ & 1.041 \\
\hline$\left[\mathrm{C}_{\mathrm{II}}\right]$ & ${ }^{2} P_{3 / 2}{ }^{2} P_{1 / 2}$ & 157.741 & 91 & $5.26 \mathrm{E}-06$ & 1.037 \\
\hline$\left[\mathrm{N}_{\mathrm{II}}\right]$ & ${ }^{3} P_{1}-{ }^{3} P_{0}$ & 205.178 & 70 & $4.51 \mathrm{E}-07$ & 1.026 \\
\hline$\left[\mathrm{CI}_{\mathrm{I}}\right]$ & ${ }^{3} P_{2}-{ }^{3} P_{1}$ & 370.414 & 63 & $1.22 \mathrm{E}-07$ & 1.013 \\
\hline$[\mathrm{C} \mathrm{I}]$ & ${ }^{3} P_{1}-{ }^{3} P_{0}$ & 609.133 & 24 & $1.95 \mathrm{E}-08$ & 1.007 \\
\hline${ }^{12} \mathrm{CO}$ & $J=24-23$ & 108.763 & 1656.6 & $1.40 \mathrm{E}-08$ & 1.058 \\
\hline${ }^{12} \mathrm{CO}$ & $J=23-22$ & 113.458 & 1524.3 & $3.49 \mathrm{E}-08^{\mathrm{d}}$ & 1.055 \\
\hline${ }^{12} \mathrm{CO}$ & $J=22-21$ & 118.581 & 1397.4 & 2.37E-08 & 1.052 \\
\hline${ }^{12} \mathrm{CO}$ & $J=21-20$ & 124.193 & 1276.1 & $3.95 \mathrm{E}-08$ & 1.049 \\
\hline${ }^{12} \mathrm{CO}$ & $J=20-19$ & 130.369 & 1160.3 & $3.58 \mathrm{E}-08$ & 1.047 \\
\hline${ }^{12} \mathrm{CO}$ & $J=19-18$ & 137.196 & 1049.9 & $5.26 \mathrm{E}-08$ & 1.044 \\
\hline${ }^{12} \mathrm{CO}$ & $J=18-17$ & 144.784 & 945.0 & 4.67E-08 & 1.041 \\
\hline${ }^{12} \mathrm{CO}$ & $J=17-16$ & 153.267 & 845.6 & 5.23E-08 & 1.038 \\
\hline${ }^{12} \mathrm{CO}$ & $J=16-15$ & 162.812 & 751.8 & 7.54E-08 & 1.035 \\
\hline${ }^{12} \mathrm{CO}$ & $J=15-14$ & 173.631 & 663.4 & $9.66 \mathrm{E}-08$ & 1.032 \\
\hline${ }^{12} \mathrm{CO}$ & $J=14-13$ & 185.999 & 580.5 & $1.30 \mathrm{E}-07$ & 1.030 \\
\hline${ }^{12} \mathrm{CO}$ & $J=13-12$ & 200.272 & 503.2 & $1.59 \mathrm{E}-07$ & 1.027 \\
\hline${ }^{12} \mathrm{CO}$ & $J=12-11$ & 216.927 & 431.3 & $1.94 \mathrm{E}-07$ & 1.025 \\
\hline${ }^{12} \mathrm{CO}$ & $J=11-10$ & 236.613 & 365.0 & $2.38 \mathrm{E}-07$ & 1.022 \\
\hline${ }^{12} \mathrm{CO}$ & $J=10-9$ & 260.240 & 304.2 & $2.75 \mathrm{E}-07$ & 1.020 \\
\hline${ }^{12} \mathrm{CO}$ & $J=9-8$ & 289.120 & 248.9 & $2.84 \mathrm{E}-07$ & 1.017 \\
\hline${ }^{12} \mathrm{CO}$ & $J=8-7$ & 325.225 & 199.1 & 3.89E-07 & 1.015 \\
\hline${ }^{12} \mathrm{CO}$ & $J=7-6$ & 371.650 & 154.9 & $3.08 \mathrm{E}-07$ & 1.013 \\
\hline${ }^{12} \mathrm{CO}$ & $J=6-5$ & 433.556 & 116.2 & $2.02 \mathrm{E}-07$ & 1.011 \\
\hline${ }^{12} \mathrm{CO}$ & $J=5-4$ & 520.231 & 83.0 & $1.34 \mathrm{E}-07$ & 1.009 \\
\hline${ }^{12} \mathrm{CO}$ & $J=4-3$ & 650.252 & 55.3 & $7.81 \mathrm{E}-08$ & 1.007 \\
\hline${ }^{13} \mathrm{CO}$ & $J=12-11$ & 226.898 & 412.4 & $6.53 \mathrm{E}-09$ & 1.023 \\
\hline${ }^{13} \mathrm{CO}$ & $J=11-10$ & 247.490 & 348.9 & $6.68 \mathrm{E}-09$ & 1.021 \\
\hline${ }^{13} \mathrm{CO}$ & $J=10-9$ & 272.205 & 290.8 & 8.52E-09 & 1.019 \\
\hline${ }^{13} \mathrm{CO}$ & $J=9-8$ & 302.415 & 237.9 & $1.94 \mathrm{E}-08$ & 1.016 \\
\hline${ }^{13} \mathrm{CO}$ & $J=8-7$ & 340.181 & 190.4 & $2.00 \mathrm{E}-08$ & 1.014 \\
\hline${ }^{13} \mathrm{CO}$ & $J=7-6$ & 388.743 & 148.1 & $1.63 \mathrm{E}-08$ & 1.012 \\
\hline${ }^{13} \mathrm{CO}$ & $J=6-5$ & 453.498 & 111.1 & $6.25 \mathrm{E}-09$ & 1.010 \\
\hline${ }^{13} \mathrm{CO}$ & $J=5-4$ & 544.161 & 79.3 & $1.08 \mathrm{E}-08$ & 1.008 \\
\hline
\end{tabular}

Notes.

a Observed lines intensities above $3 \sigma$ in $\mathrm{W} \mathrm{m}^{-2} \mathrm{sr}^{-1}$. Absolute calibration accuracy up to $\sim 30 \%$.

${ }^{\mathrm{b}}$ Extinction correction factors.

${ }^{c}$ From Shields \& Ferland (1994).

${ }^{\mathrm{d}}$ Blended with the $\mathrm{o}-\mathrm{H}_{2} \mathrm{O} 4_{14}-3_{03}$ line.

\subsection{Ionized Gas and Neutral Atomic Gas}

The velocity resolution of PACS spectra at short wavelengths, $\lesssim 100 \mathrm{~km} \mathrm{~s}^{-1}$, allows one to resolve shifts in the line profile peak velocity if they are large. Figures 1(c) and (d) show the [OI] 63 and [N III] $57 \mu \mathrm{m}$ line maps. The Doppler shifts of both lines reveal motions of the neutral and of the ionized gas, with blueshifted velocities observed toward the $S$-CND and redshifted toward the $N$-CND. The [N III] $57 \mu \mathrm{m}$ lines show a similar pattern to the [Ne II] $13 \mu \mathrm{m}$ lines observed at higher spatial and spectral resolution (Irons et al. 2012). They are consistent with ionized gas streamers orbiting or falling in a potential dominated by the central black hole (Serabyn \& Lacy 1985; Paumard et al. 2004). Note that both the [O I] 63 and [N III] $57 \mu \mathrm{m}$ lines show high-velocity wing emission ( $\gtrsim \pm 300 \mathrm{~km} \mathrm{~s}^{-1}$ ) toward Sgr A* and narrower profiles at greater distances from the center. This high-velocity gas is likely associated with clouds/clumps moving fast and close to Sgr A*.

The spatial distribution of the [N III] lines follows the dense filaments $\left(n_{\mathrm{e}}>10^{3.5} \mathrm{~cm}^{-3}\right)$ of ionized gas orbiting Sgr A* (the mini-spiral shown in Figure 1; Yusef-Zadeh \& Morris 1987). This lower limit to the electron density $\left(n_{\mathrm{e}}\right)$ is estimated by comparing the [O III] 88-to-[O III]-52 line intensity ratio of $0.21 \pm 0.12$ (hereafter [O III] 88/52) with the semi-empirical prescription of Rubin et al. (1994).

The [N III] 57 and [N II] $122 \mu \mathrm{m}$ line intensities can also be used to estimate the effective temperature of the ionizing radiation $\left(T_{\text {eff }}\right)$ for a given $n_{\mathrm{e}}$ (Rubin et al. 1994). The [N III] 57/ [N II] $122=1.31 \pm 0.51$ intensity ratio toward $\mathrm{Sgr} \mathrm{A}^{*}$ results in $T_{\text {eff }} \simeq 35,000 \pm 1000 \mathrm{~K}$ (see Shields \& Ferland 1994 for photoionization detailed models).

The strong UV radiation field from stars in the central parsec is thought to dominate the heating of the dust grains and of the neutral atomic gas (Genzel et al. 1985; Jackson et al. 1993). The low $\left(L_{[\mathrm{OI}]}+L_{[\mathrm{CII}]}\right) / L_{\mathrm{FIR}} \simeq 8 \times 10^{-3}$ luminosity ratio toward the central cavity agrees with the low efficiency heating mechanisms expected in photodissociation regions (PDRs). In addition, [O I] 63/[C II] $158=3.32 \pm 1.31$ and [O I ] 145/63= $0.12 \pm 0.05$ line intensity ratios are observed toward Sgr $\mathrm{A}^{*}$. These are similar to the ratios observed in strongly irradiated PDRs like the Orion Bar (Bernard-Salas et al. 2012) but are significantly lower than the expected ratios in shocked gas and in X-ray-dominated regions (XDRs; Maloney et al. 1996). Therefore, the neutral atomic gas $\left(n_{\mathrm{H}} \sim 10^{4-5} \mathrm{~cm}^{-3}\right)$ is predominantly heated by UV photons $\left(G_{0}>10^{4}\right.$ times the mean interstellar radiation field). Atomic gas temperatures $\lesssim 500 \mathrm{~K}$ are expected in this PDR range (Wolfire et al. 1990). Nevertheless, narrow (unresolved) absorption components from foreground gas in the GC (e.g., Sonnentrucker et al. 2013) can affect the total [O I] 63 and [C II] $158 \mu \mathrm{m}$ fluxes measured by PACS at medium spectral resolution (both are ground-state transitions). Hence, the actual ratios may be different, and our unresolved $[\mathrm{O} \mathrm{I}]$ and $[\mathrm{C} \mathrm{II}]$ intensities may have less diagnostic power.

\subsection{Hot Molecular Gas}

Figure 3(a) shows all detected ${ }^{12} \mathrm{CO}$ lines toward the central cavity on a rotational diagram that assumes extended emission. The average ${ }^{12} \mathrm{CO} /{ }^{13} \mathrm{CO}$ line intensity ratio is $22 \pm 9$ (lines $J=$ 5-4 to 10-9), thus consistent with the ${ }^{12} \mathrm{C} /{ }^{13} \mathrm{C} \simeq 20-25$ isotopic ratio inferred in the Sgr A complex (Penzias 1980). Hence, the observed line intensity ratios are compatible with optically thin ${ }^{12} \mathrm{CO}$ line emission (see the next section).

By fitting the ${ }^{12} \mathrm{CO}$ lines detected by SPIRE and PACS independently, we obtain $T_{\text {rot }}(\mathrm{SPIRE}) \simeq 87 \mathrm{~K}$ and $T_{\text {rot }}(\mathrm{PACS}) \simeq$ $232 \mathrm{~K}$, respectively. $T_{\text {rot }}$ is a good measure of the gas temperature only in the high-density limit (close to local thermodynamic equilibrium, LTE). In this case, the two slopes could be associated with two different temperature components, the hotter one representing $\lesssim 4 \%$ of the total ${ }^{12} \mathrm{CO}$ column density. Alternatively, $T_{\text {rot }}$ can reflect much higher gas temperatures if the density is significantly lower than the critical density for collisional excitation $\left(n_{\mathrm{cr}}\left(\mathrm{H}_{2}\right)>10^{7} \mathrm{~cm}^{-3}\right.$ for the observed high- $J$ lines).

In fact, a closer inspection of the rotational diagram shows that $T_{\text {rot }}$ increases with $J$, from $T_{\text {rot }}(14-18) \simeq 160 \mathrm{~K}$ to $T_{\text {rot }}(19-24) \simeq 250 \mathrm{~K}$. Therefore, the rotational diagram shows a moderate positive curvature and thus even a single-temperature 

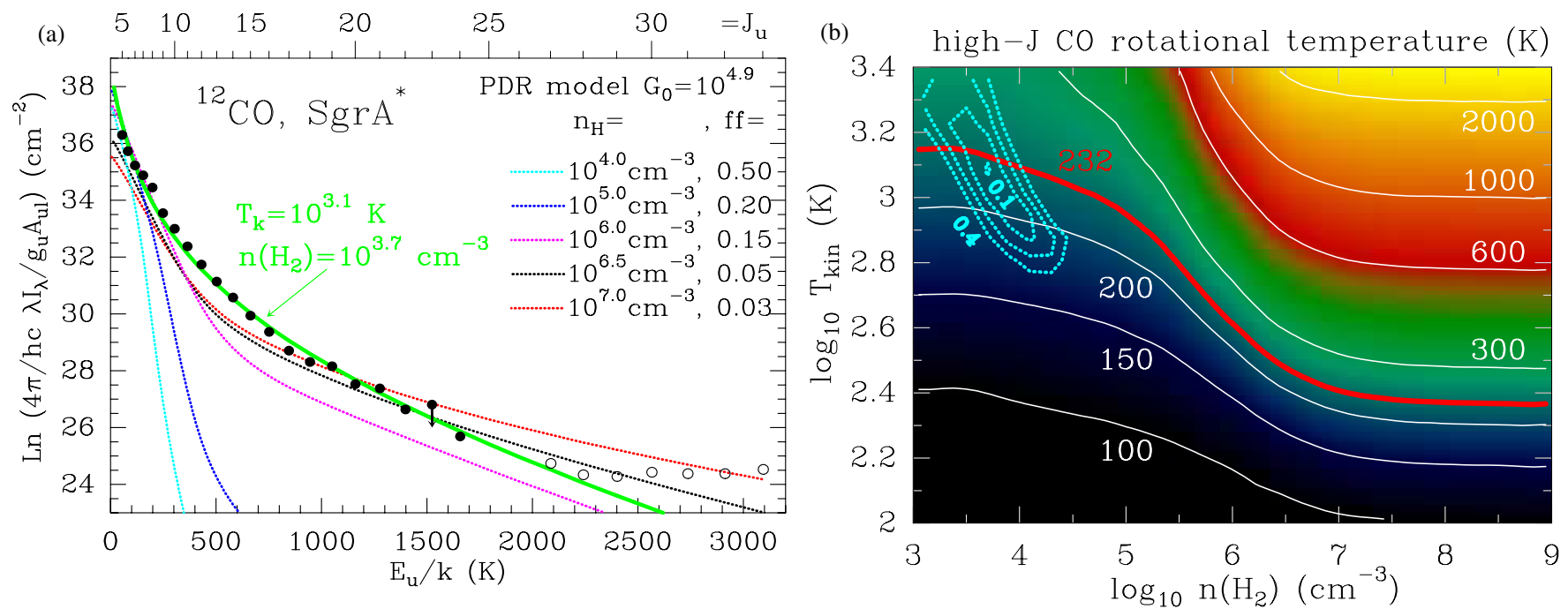

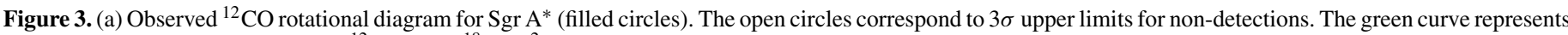

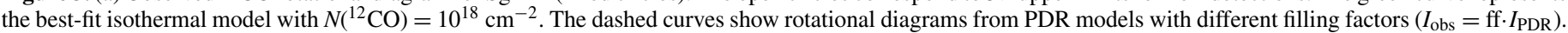

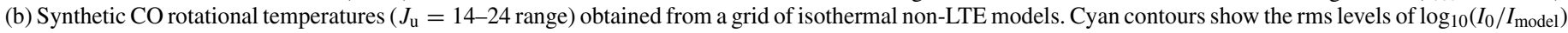
for fits to the absolute line intensities from 0.1 (best fits with an rms error of $\sim 25 \%$ ) to 0.4 in rms error steps of 0.1 .

(A color version of this figure is available in the online journal.)

component in the LTE cannot explain the $\mathrm{CO}$ emission detected by PACS. Neufeld (2012) pointed out that a moderate positive curvature can be explained either by (1) a subthermally excited single-temperature component $\left(T_{\mathrm{k}} \gg T_{\text {rot }}\right)$ or (2) multipletemperature components (that could be in LTE).

In order to constrain the range of physical conditions that reproduce the observed $\mathrm{CO}$ intensities, we have run a grid of non-local, non-LTE isothermal models (Goicoechea et al. 2006) using ${ }^{12} \mathrm{CO}-\mathrm{H}_{2}$ collisional rates from Yang et al. (2010). We used a constant beam-averaged $\mathrm{CO}$ column density, $N(\mathrm{CO})$, and a non-thermal velocity dispersion $\sigma=65 \mathrm{~km} \mathrm{~s}^{-1}$ (from turbulence and macroscopic gas motions), implying $\Delta v \simeq 150 \mathrm{~km} \mathrm{~s}^{-1}$ line widths. These broad widths are consistent with the mid$J$ CO line widths observed with Herschel/HIFI (T.A. Bell 2013, private communication). We adopt $N(\mathrm{CO})=\chi(\mathrm{CO}) \times$ $N\left(\mathrm{H}_{2}\right)=10^{18} \mathrm{~cm}^{-2}$ (Genzel et al. 1985), i.e., we assume a CO abundance of $\simeq 10^{-4}$ and take, from photometric measurements, $N\left(\mathrm{H}_{2}\right) \simeq 10^{22} \mathrm{~cm}^{-2}\left(A_{V} \sim 10\right)$ in the central cavity (Etxaluze et al. 2011).

Figure 3(b) shows the model results in the form of iso- $T_{\text {rot }}$ contours. To make this plot, we first created rotational diagrams from each model and determined $T_{\text {rot }}$ by fitting a straight line to the synthetic CO line intensities in the $J_{\text {up }}=16-24$ range. Figure 3(b) shows that in terms of excitation alone the same $T_{\text {rot }}(\mathrm{PACS}) \approx 232 \mathrm{~K}$ can be obtained for different combinations of density and temperature. In a second step, we searched for the range of $n\left(\mathrm{H}_{2}\right)$ and $T_{\mathrm{k}}$ values that better reproduce the observed ${ }^{12} \mathrm{CO}$ lines by fitting their absolute intensities. For the adopted $N(\mathrm{CO})$, the best-fit parameters are obtained around $T_{\mathrm{k}} \simeq 10^{3.1} \mathrm{~K}$ and $n\left(\mathrm{H}_{2}\right) \simeq 10^{3.7} \mathrm{~cm}^{-2}$. The resulting best-fit rotational diagram is shown as a green curve in Figure 3(a) (note that it is also consistent with the $3 \sigma$ upper limits for higher- $J$ undetected lines).

In a third step, we compared the observations with the more extensive model grid of Neufeld (2012) and studied the dependence of our results on the assumed $N(\mathrm{CO})$. For isothermal models, the gas temperature is well constrained and $T_{\mathrm{k}} \simeq 10^{3.1} \mathrm{~K}$ reproduces the observed $\mathrm{CO}$ rotational ladder (also the lines detected by PACS alone) independently of the assumed $N(\mathrm{CO})$. Even lower densities (for less realistic higher $N(\mathrm{CO})$ columns) can also reproduce the $\mathrm{CO}$ ladder.

In addition to the isothermal solution, multiple gas temperature components can also explain a $\mathrm{CO}$ rotational diagram with positive curvature. Neufeld (2012) studied the case of a medium with a power-law distribution of temperatures, $d N(\mathrm{CO}) / d T_{\mathrm{k}}=a T_{\mathrm{k}}^{-b}$. Including all CO lines observed by Herschel in the fit gives $b \simeq 2.0-2.5$ and $n\left(\mathrm{H}_{2}\right) \simeq 10^{4-5} \mathrm{~cm}^{-3}$. In other words, although a small fraction of the total ${ }^{12} \mathrm{CO}$ column exists at $T_{\mathrm{k}}>300 \mathrm{~K}(\lesssim 4 \%)$, most of the $N(\mathrm{CO})$ column will be at lower temperatures and higher densities than those implied by the isothermal solution. We therefore conclude that the observed $\mathrm{CO}$ lines are consistent with either a single, hot $\left(T_{\mathrm{k}} \simeq 10^{3.1} \mathrm{~K}\right)$, low-density $\left(n\left(\mathrm{H}_{2}\right) \lesssim 10^{4} \mathrm{~cm}^{-3}\right)$ component or with multiple, cooler components at a higher density. In the latter case, the required density will be above the beam-averaged gas densities in the central cavity $\left(\sim 10^{3-4} \mathrm{~cm}^{-3}\right.$; Etxaluze et al. 2011), implying that the hot molecular gas in the vicinity of Sgr A* does not have a homogeneous distribution but fills a small fraction of the volume.

\section{DISCUSSION}

In this section we discuss the possible heating mechanisms of the hot molecular gas toward the central parsec. In order to evaluate the role of $U V$ radiation in the heating and excitation of CO, we used an updated version of the Meudon PDR code (Le Bourlot et al. 2012) to compute synthetic CO rotational diagrams for the integrated $\mathrm{CO}$ emission from $A_{\mathrm{V}}=0$ to 10 . We adopted $G_{0}=10^{4.9}$ (Wolfire et al. 1990) and different gas densities. Our photochemical model includes selective photodissociation of $\mathrm{CO}$ isotopologues and ${ }^{13} \mathrm{C}$ fractionation. For the considered range of densities, selective photodissociation slightly increases the ${ }^{12} \mathrm{CO} /{ }^{13} \mathrm{CO}$ column density ratio over the ${ }^{12} \mathrm{C} /{ }^{13} \mathrm{C}$ isotopic ratio (by $\lesssim 25 \%$ ) at the $A_{\mathrm{V}}<2$ surface layers where CO columns are still low. Deeper inside, as the gas temperature decreases, ${ }^{13} \mathrm{C}$ isotope exchange starts to be important and the ${ }^{12} \mathrm{CO} /{ }^{13} \mathrm{CO}$ column density ratio can be lower than the ${ }^{12} \mathrm{C} /{ }^{13} \mathrm{C}$ ratio. All in all, we conclude that the beam-averaged $N\left({ }^{12} \mathrm{CO}\right)$ toward Sgr A* 
cannot be much larger than the adopted $\sim 10^{18} \mathrm{~cm}^{-2}$. For these columns and large velocity dispersions, the observed ${ }^{12} \mathrm{CO}$ lines are optically thin. Figure 3(a) shows the resulting $\mathrm{CO}$ diagrams for different PDR models and filling factors. By comparing with observations, we see that low-density PDRs $\left(n_{\mathrm{H}} \leqslant 10^{5} \mathrm{~cm}^{-3}\right)$ are not able to reproduce the high- $J$ CO emission and an extra heating/excitation source is needed. In addition to photoelectric heating, denser PDRs $\left(n_{\mathrm{H}} \simeq 10^{6-7} \mathrm{~cm}^{-3}\right)$ heat larger columns of molecular gas (to $T_{k} \sim 10^{3} \mathrm{~K}$ ) by vibrational heating from collisional de-excitation of UV-pumped $\mathrm{H}_{2}$ molecules. Therefore, in addition to an extended low-density medium, a small filling factor ensemble of irradiated dense clumps/ clouds could be responsible of the high- $J$ CO emission (Burton et al. 1990). Our best-combined PDR models, however, do not provide an entirely satisfactory fit of the $\mathrm{CO}$ rotational ladder. This result is consistent with the lack of good high-density fits to the high- $J$ CO lines (see the previous section) and suggests that UV radiation alone cannot heat the hot molecular gas.

Interestingly, the SPIRE-FTS spectrum toward Sgr A* resembles that of the M82 starburst galaxy (Kamenetzky et al. 2012). However, the hot $\mathrm{CO}$ rotational temperatures inferred toward Sgr A*are significantly higher than those seen in strongly irradiated PDRs like the Orion Bar, which shows a rotational diagram (up to $J=21-20$ ) that can be fitted with a single $T_{\text {rot }}(\mathrm{CO}) \simeq 150 \mathrm{~K}$ component (Habart et al. 2010; C. Joblin et al., in preparation). In addition, even toward the strongly UV-irradiated central cavity, the observed $L(\mathrm{CO}) / L_{\mathrm{FIR}} \simeq 10^{-3}$ luminosity ratio is higher than the expected ratio in PDRs and XDRs models (Meijerink et al. 2013), and it is indeed higher than the observed value in the Orion $\operatorname{Bar}\left(L(\mathrm{CO}) / L_{\mathrm{FIR}} \simeq\right.$ $3 \times 10^{-4}$; C. Joblin 2013, private communication).

The current X-ray luminosity near $\operatorname{Sgr} \mathrm{A}^{*}$ is rather low $\left(L_{\mathrm{X}}(2-120 \mathrm{keV})<10^{36} \mathrm{erg} \mathrm{s}^{-1}\right.$; Bélanger et al. 2006), far lower than that expected from black hole accretion models. Hence, any incident X-ray flux at a typical distance of $\sim 0.5 \mathrm{pc}$ from the source $\left(F_{\mathrm{X}}<0.03 \mathrm{erg} \mathrm{cm} \mathrm{cm}^{-2} \mathrm{~s}^{-1}\right)$ would be too low to heat a significant fraction of the molecular gas well above $T_{\mathrm{k}} \sim 100 \mathrm{~K}$ (Maloney et al. 1996).

High cosmic-ray $(\mathrm{CR})$ ionization rates $\left(\zeta_{\mathrm{CR}} \gtrsim 10^{-15} \mathrm{~s}^{-1}\right)$ have been inferred in the $\mathrm{GC}$ region from $\mathrm{H}_{3}^{+}$observations, and even higher rates have been proposed for the vicinity of Sgr A* $\left(\sim 2 \times 10^{-14} \mathrm{~s}^{-1}\right.$; Goto et al. 2008). At least qualitatively, our detection of $\mathrm{H}_{3} \mathrm{O}^{+}$absorption lines from excited metastable levels indeed suggests that $\zeta_{\mathrm{CR}}$ may be high. Nevertheless, the ionization fraction of the molecular gas in the central parsec seems lower than that in much more extreme X-ray dominated AGNs like Mrk $231\left(L_{\mathrm{X}}(2-10 \mathrm{keV}) \simeq 6 \times 10^{43} \mathrm{erg} \mathrm{s}^{-1}\right)$, where strong $\mathrm{CH}^{+}, \mathrm{OH}^{+}$, and $\mathrm{H}_{2} \mathrm{O}^{+}$emission lines have been detected (van der Werf et al. 2010). It also has to be lower than in the ULIRG galaxy Arp 220, where the detection of many excited $\mathrm{OH}^{+}$and $\mathrm{H}_{2} \mathrm{O}^{+}$absorption lines has been associated with very enhanced $\mathrm{X}$-ray/CR ionization rates $\left(\zeta_{\mathrm{X}, \mathrm{CR}}>10^{-13} \mathrm{~s}^{-1}\right.$; González-Alfonso et al. 2013). Our observations toward Sgr A* show that $\mathrm{OH}^{+}$and $\mathrm{H}_{2} \mathrm{O}^{+}$only produce appreciable groundstate absorption lines, and they are known to arise from semiatomic diffuse clouds, where their columns are proportional to $\zeta_{\mathrm{CR}}$ (Gerin et al. 2010; Neufeld et al. 2010; Hollenbach et al. 2012). Hence, $\zeta_{\mathrm{CR}}$ toward Sgr $A^{*}$ is very likely higher than in Galactic disk clouds, but lower than $\zeta_{\mathrm{X}, \mathrm{CR}}$ in Mrk 231 or Arp 220. Simple thermodynamic considerations show that even $\zeta_{\mathrm{CR}}=2 \times 10^{-14} \mathrm{~s}^{-1}$ would only heat the gas to a few tens of $\mathrm{K}$ (see also Glassgold et al. 2012). Therefore, neither X-rays nor
CRs presently dominate the heating of the hot molecular gas near Sgr A*.

Low-density shocks (and related supersonic turbulence dissipation and magnetic viscous heating) are promising candidates in the highly magnetized GC environment (Morris \& Serabyn 1996). In particular, non-dissociative, magnetohydrodynamic shocks tend to produce regions that are roughly isothermal, reaching very high temperatures without destroying molecules $\left(T_{\mathrm{k}}>1000 \mathrm{~K}\right.$ for shock velocities $v_{\mathrm{s}}>20 \mathrm{~km} \mathrm{~s}^{-1}$ in C-type shock models by Kaufman \& Neufeld 1996). In fact, shocks with a variety of densities, $v_{s}$, and magnetic field strengths dominate the heating of the hot molecular gas seen in protostellar outflows (with $L(\mathrm{CO}) / L_{\mathrm{FIR}} \simeq 2 \times 10^{-3}$ observed in Serpens SMM1; Goicoechea et al. 2012). Hence, the hot $\mathrm{CO}$ gas inferred toward Sgr A*, the high $L(\mathrm{CO}) / L_{\mathrm{FIR}}$ ratio, and the almost thermal $\mathrm{H}_{2}$ rovibrational spectrum (Tanaka et al. 1989) suggest that, in addition to UV-driven excitation, shocks contribute to the heating of the hot molecular gas in the nucleus of the Galaxy. Indeed, if a small filling factor ensemble of dense clumps/clouds does not exist, shocks likely dominate.

Whether the required shocks are produced within highvelocity molecular gas falling toward the very center region (Gillessen et al. 2012) arise in clump-clump collisions (MartínPintado et al. 1997), or in outflows driven by high-velocity stellar winds (Najarro et al. 1997), or by protostars in the central parsec (Nishiyama \& Schödel 2013) is still uncertain.

We thank C. Lang and M. A. Requena-Torres for providing us with the VLA $6 \mathrm{~cm}$ radiocontinuum image in CLASS format, and $\mathrm{C}$. Joblin and $\mathrm{O}$. Bernè for useful discussions on the Orion Bar. We thank the Spanish MINECO for funding support from grants AYA2009-07304, CSD2009-00038, and S2009ESP-1496, and NASA through an award issued by JPL/ Caltech. J.R.G. is supported by a Ramón y Cajal research contract.

\section{REFERENCES}

Bélanger, G., Glowworm, A., Renaud, M., et al. 2006, ApJ, 636, 275 Bernard-Salas, J., Habart, E., Arab, H., et al. 2012, A\&A, 538, A37 Bradford, C. M., Stacey, G. J., Nikola, T., et al. 2005, ApJ, 623, 866 Burton, M. G., Hollenbach, D. J., \& Tielens, A. G. G. M. 1990, ApJ, 365, 620 Etxaluze, M., Smith, H. A., Tolls, V., Stark, A. A., \& González-Alfonso, E. 2011, AJ, 142, 134

Genzel, R., Crawford, M. K., Townes, C. H., \& Watson, D. M. 1985, ApJ, 297, 766

Genzel, R., Eisenhauer, F., \& Gillessen, S. 2010, RvMP, 82, 3121

Gerin, M., de Luca, M., Black, J., et al. 2010, A\&A, 518, L110

Gillessen, S., Genzel, R., Fritz, T. K., et al. 2012, Natur, 481, 51

Glassgold, A. E., Galli, D., \& Padovani, M. 2012, ApJ, 756, 157

Goicoechea, J. R., Cernicharo, J., Karska, A., et al. 2012, A\&A, 548, A77

Goicoechea, J. R., Pety, J., Gerin, M., et al. 2006, A\&A, 456, 565

González-Alfonso, E., Fischer, J., Bruderer, S., et al. 2013, A\&A, 550, 25

Goto, M., Usuda, T., Nagata, T., et al. 2008, ApJ, 688, 306

Griffin, M. J., Abergel, A., Abreu, A., et al. 2010, A\&A, 518, L3

Guesten, R., Genzel, R., Wright, M. C. H., et al. 1987, ApJ, 318, 124

Habart, E., Dartois, E., Abergel, A., et al. 2010, A\&A, 518, L116

Herrnstein, R. M., \& Ho, P. T. P. 2002, ApJL, 579, L83

Hollenbach, D., Kaufman, M. J., Neufeld, D., Wolfire, M., \& Goicoechea, J. R. 2012, ApJ, 754, 105

Irons, W. T., Lacy, J. H., \& Richter, M. J. 2012, ApJ, 755, 90

Jackson, J. M., Geis, N., Genzel, R., et al. 1993, ApJ, 402, 173

Kamenetzky, J., Glenn, J., Rangwala, N., et al. 2012, ApJ, 753, 70

Kaufman, M. J., \& Neufeld, D. A. 1996, ApJ, 456, 611

Le Bourlot, J., Le Petit, F., Pinto, C., Roueff, E., \& Roy, F. 2012, A\&A, 541, A76

Lutz, D. 1999, in The Universe as Seen by ISO, ed. P. Cox \& M. F. Kessler (ESA SP-427; Noordwijk: European Space Agency), 623 
Maloney, P. R., Hollenbach, D. J., \& Tielens, A. G. G. M. 1996, ApJ, 466, 561 Martín-Pintado, J., de Vicente, P., Fuente, A., \& Planesas, P. 1997, ApJL, 482, L45

Meijerink, R., Kristensen, L. E., Weiß, A., et al. 2013, ApJL, 762, L16

Moneti, A., Cernicharo, J., \& Pardo, J. R. 2001, ApJL, 549, L203

Montero-Castaño, M., Herrnstein, R. M., \& Ho, P. T. P. 2009, ApJ, 695, 1477

Morris, M., \& Serabyn, E. 1996, ARA\&A, 34, 645

Najarro, F., Krabbe, A., Genzel, R., et al. 1997, A\&A, 325, 700

Neufeld, D. A. 2012, ApJ, 749, 125

Neufeld, D. A., Goicoechea, J. R., Sonnentrucker, P., et al. 2010, A\&A, 521, L10

Nishiyama, S., \& Schödel, R. 2013, A\&A, 549, A57

Paumard, T., Maillard, J.-P., \& Morris, M. 2004, A\&A, 426, 81

Penzias, A. A. 1980, Sci, 208, 663
Pilbratt, G. L., Riedinger, J. R., Passvogel, T., et al. 2010, A\&A, 518, L1 Poglitsch, A., Waelkens, C., Geis, N., et al. 2010, A\&A, 518, L2 Reid, M. J. 1993, ARA\&A, 31, 345

Requena-Torres, M. A., Güsten, R., Weiß, A., et al. 2012, A\&A, 542, L21

Rubin, R. H., Simpson, J. P., Lord, S. D., et al. 1994, ApJ, 420, 772

Serabyn, E., \& Lacy, J. H. 1985, ApJ, 293, 445

Shields, J. C., \& Ferland, G. J. 1994, ApJ, 430, 236

Sonnentrucker, P., Neufeld, D. A., Gerin, M., et al. 2013, ApJL, 763, L19

Tanaka, M., Hasegawa, T., Hayashi, S. S., Brand, P. W. J. L., \& Gatley, I. 1989, ApJ, 336, 207

van der Werf, P. P., Isaak, K. G., Meijerink, R., et al. 2010, A\&A, 518, L42

Wolfire, M. G., Tielens, A. G. G. M., \& Hollenbach, D. 1990, ApJ, 358, 116

Yang, B., Stancil, P. C., Balakrishnan, N., \& Forrey, R. C. 2010, ApJ, 718, 1062

Yusef-Zadeh, F., \& Morris, M. 1987, ApJ, 320, 545 